Center

for

Economic Research

No. $2000-57$

INCREASING RETURNS AND CYCLES IN

FISHING

By Matti Liski, Peter M. Kort and Andreas Novak

June 2000

ISSN 0924-7815 


\title{
Increasing Returns and Cycles in Fishing
}

\author{
Matti Liski ${ }^{a}$, Peter M. Kort ${ }^{b}$ and Andreas Novak $^{c}$
}

May 19, 2000

\begin{abstract}
We consider optimal fishery management under the assumption of increasing returns that is supported by previous empirical evidence. We improve the tractability and realism of the previous approaches by introducing flow adjustment costs on changes in harvest rate. Our framework is the first to provide a link between stable limit cycle policies and increasing returns in harvesting. The type of the harvest policy depends on flow adjustment costs: for relatively costly adjustments the usual steady state harvest policy is conceivable, whereas for relatively cheap adjustments the harvest policy is cyclical. We also show a connection between chattering control policies and limit cycles, which helps us to develop a clear economic meaning for cyclical harvesting. Keywords: Nonconvexities; Adjustment costs; Hopf bifurcation; Renewable resources. JEL classification:C61;C62.

${ }^{a}$ corresponding author, Helsinki School of Economics/P.O. Box 1210/00101 Helsinki/ Finland/liski@hkkk.fi

${ }^{b}$ Department of Econometrics and CentER/TilburgUniversity/5000 LE Tilburg/The Netherlands/kort@kub.nl

${ }^{c}$ Department of Statistics and Decision Support Systems, University of Vienna, 1010 Vienna/Austria/ andreas.novak@univie.ac.at
\end{abstract}




\section{Introduction}

The possibility of increasing returns in fishery management is relevant both from the empirical and theoretical point of view. Recently, [1] estimated a production function for the Norwegian Seal hunt based on time series data. The results indicated increasing returns with respect to the number of boats on the fishing ground, "which may be due to boats sharing information about locations of seals" (p. 165). [2] undertook a similar type of estimation for the North Sea Herring fishery and concluded that "the hypothesis of increasing returns in all factors is generally accepted". Similar results were obtained earlier by [11] for the North Atlantic Cod fishery. Theoretically, the case is interesting because the assumption of increasing returns in harvesting can dramatically change the implications of the standard fishery management model. Harvesting strategies that stabilize the stock of fish to the usual steady state level may be replaced by policies involving the abandonment [15] or cyclical utilization of the resource $([16],[3],[6])$.

Perhaps the most insightful implication of this previous theoretical literature is the possibility that cyclical harvesting policies can be optimal. Increasing returns could thus potentially explain "pulse fishing" that is frequently observed in practice $[3]^{1}$. The economic meaning of cycles can be related to U-shaped average harvest costs stipulating a minimum efficient scale of harvest. If this critical harvest rate is too large to stabilize the stock, the usual steady state harvesting policy becomes infeasible. Instead, fishing fleets should leave and re-enter the fishing ground regularly such that the stock suitably recovers from excessive harvesting during each inactive fishing period. The stock is in this way stabilized over time. ${ }^{2}$

\footnotetext{
${ }^{1}$ In [1], the data covering the years 1952-1972 show a relatively regular cyclical pattern for the harvest. Further examples are discussed in [16].

${ }^{2}$ In a multi-cohort fishery, the reasons for pulse fishing can be related to the different economic valuation of various year classes [11].
} 
While the above insight is valuable, increasing returns can jeopardize the usefulness of the standard fishery management model as a tool for evaluating and explaining the harvest policies occurring in practice. The optimal harvest policy can be characterized by extremely short cycles, meaning that the fleets should leave and re-enter the fishing ground almost uninterruptedly $([3],[16])$. In the theoretical literature such harvest policies are called chattering strategies. This theoretical implication is unreasonable since it is driven by the assumption that the fishery manager can completely avoid the cost flow due to investments in vessels, fishing equipments, and to hiring and training workers by temporally shutting down the fishery. In practice, it is virtually impossible to instantaneously buy and sell vessels, move the fleet from one fishery to another or hire and fire workers. Such difficulties indicate the presence of adjustment costs. These costs arise, for example, since the fleet must be sold, scrapped or moved to another fishing ground when the fishery is inactive. Resources must also be committed to rehiring and retraining of labor if pulse fishing is accompanied by layoffs.

The previous literature has incorporated adjustment costs in the form of start-up or re-entry costs incurred whenever harvesting is resumed after an inactive period ([15],[16]). These recurring one-time payments may produce a more reasonable implication: the fishery may go from a possibly long period of no harvesting to a period of active harvesting. But this approach should be viewed only as the first approximation of adjustment costs because the harvest rate and start-up costs are independent of each other. The cyclical utilization is still characterized by complete and temporary shutdowns during which all costs from the fishing activity are avoided. Perhaps more importantly, U-shaped average harvest costs accompanied by start-up costs can jeopardize the tractability of the fishery management model [16].

We suggest a simple way of improving the realism and tractability of the fishery manage- 
ment model under increasing returns. Instead of thinking harvest as a flow and adjustment cost as a stock, we replace the dimensions of these variables: the harvest level is of a stock and adjustment cost of a flow dimension. The current harvest is determined by past decisions to move resources to or away from the fishing activity, for example, by past changes in the number of vessels or fishermen. Given these past decisions, the cost flow due to the fishing capacity is not avoidable overnight but can be altered by gradually adjusting the harvest level. These adjustments incur flow costs arising, for example, in the process of hiring an additional fisherman or purchasing a vessel. In this way the harvest level and adjustment costs depend on each other.

The first advantage of our framework is that we can explicitly show the existence of two types of possible optimal policies depending on the size of adjustment costs. In case these costs are of considerable size, there exists a harvest policy that will eventually stabilize the stock of fish to a steady state level. For relatively modest adjustment costs, all conceivable policies will exhibit a cyclical movement over time. In particular, we can explicitly show the existence and stability of a limit cycle policy. The result indicates that flow adjustment costs need not alter the cyclicity of the harvest rate that prevails when adjustments are costless. It also provides a new manifestation of pulse fishing under increasing returns: the cyclical utilization of the resource involves smooth fluctuations rather than extreme shutdowns of the fishery. To our knowledge, the possibility of limit cycles in connection with renewable resource use under increasing returns has not been previously addressed.

The second advantage of our approach is that we can provide a clear economic meaning for the limit cycle. In our model, a limit cycle can exist only if the harvest policy is chattering when the cost of adjusting the harvest rate is absent. Using this connection, the economic meaning of limit cycles follows readily. When harvesting is best characterized by chattering, it is profitable to take full advantage of increasing returns by temporally harvesting excessive 
quantities and to stabilize the stock of fish over time by cutting the harvest rate back after each period of excessive harvesting. In the presence of relatively low adjustment costs, a limit cycle presents a 'sluggish' chattering policy whose economic meaning is explained, as in the case of chattering, by the presence of increasing returns. However, if these adjustment costs are large it is expensive to have a nonconstant harvest rate, so that saddlepoint stability with a constant harvest rate becomes more attractive. This would occur in situations where high hiring/firing of workers is costly, or if large investment expenses are needed to acquire new fishing boats.

Another explanation for the regularly occurrence of pulse fishing in practice is provided by [19], who considers a model that is a variant of the widely quoted model in [4]. Here the occurrence of a cyclical harvest policy is caused by the presence of a positive externality from the stock of fish (this result is also obtained in [17], in which a simplified version of ([19]) is considered). Remarkably enough, and contrary to our results, in [19] saddlepoint stability for low adjustment costs is found (in the context of the Ramsey model, a similar result is obtained in [20], p.319). Unfortunately, the intuition for such a result is not provided.

Section 2 incorporates increasing returns into the basic fishing model and explains the unimplementable chattering control solution. In Section 3, we prove the existence of an optimal solution and limit cycles when the basic model is extended by introducing adjustment costs on changing the harvest. Section 4 demonstrates the stability of a limit cycle. Concluding remarks are given in Section 5.

\section{Fishery Model with Chattering Harvest}

Bioeconomic theory tells us that in an open-access fishery rents tend to dissipate and stocks may be extincted. These findings have support in real life. To prevent overfishing and 
possible depletion of stocks, considerable efforts have been devoted to the development of appropriate means of regulation. One means of regulation is to give sole-owner rights to a single firm. Since the present and future performance of this firm depends on the resource stock, incentives and responsibility for sustainable resource use may be established. Trends of converting common property fisheries into single-owner fisheries have been seen in Canada, New Zealand, Finland and Iceland [17].

In this and the next section single-owner fishery models are developed, with the aim to study optimal harvesting policies in the presence of increasing returns. We first consider the following fishery model:

$$
\begin{gathered}
\max _{y} \int_{0}^{\infty}\{p y-c(y)\} e^{-\rho t} d t, \\
\dot{x}=F(x)-y, x(0)=x_{0},
\end{gathered}
$$

where $y$ is the harvest rate, $p$ is the constant price of fish, $c(y)$ is the cost of harvesting, $x$ is the stock of fish, $F(x) \equiv s(\bar{x}-x) x$ is the logistic growth of the stock, and $\rho$ is the constant discount rate. While this simple formalization ignores many important aspects of the fishing activity, it provides a useful framework for explaining the economic meaning of cycles caused by increasing returns; these insights will not be lost as the model is extended. To keep later expressions parsimonious in parameters, we assume that $s=\bar{x}=1>\rho$. As argued in [13], the price taker assumption does not necessarily conflict with the assumption of single ownership of the resource. We might think of the stock of resource as of many noninteracting stocks whose harvests together form a single world market for this fish. To avoid the complication of multiple steady states, we assume throughout the paper that the marginal revenue remains positive when the maximum sustainable yield is harvested, i.e., $p-c^{\prime}\left(y_{m s y}\right)>0$ where $y_{m s y} \equiv \max F(x)$.

Average harvest costs are U-shaped which implies initial increasing returns in harvesting. 


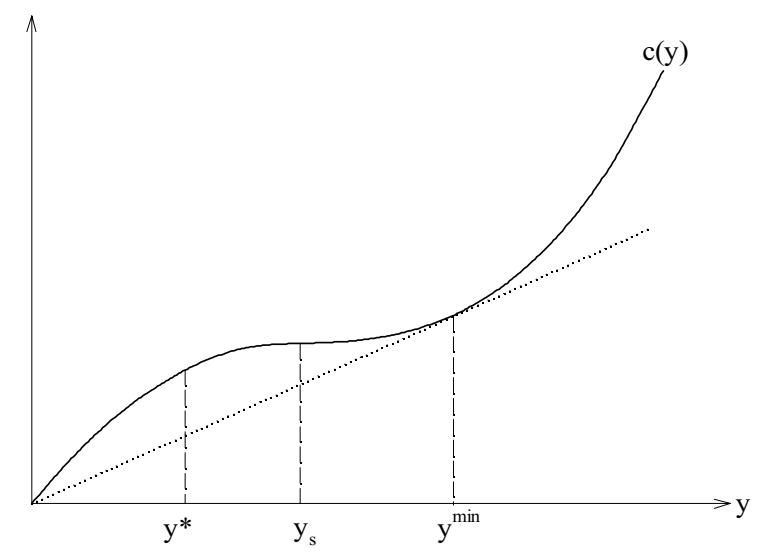

Figure 1: Harvest Cost with Initial Concavity

A way to formalize this is to assume an initial concavity in the cost function: $c^{\prime}(y)>0$ and

$$
\begin{aligned}
& c^{\prime \prime}(y)<0 \text { for } y<y_{s}, \\
& c^{\prime \prime}(y)>0 \text { for } y>y_{s} .
\end{aligned}
$$

The cost function is depicted in fig. 1 where the minimum efficient scale of fishing is denoted by $y^{\mathrm{min}}$. Initially decreasing average harvest costs may reflect the fact that some cost components are to a large extent independent of the harvest rate, including costs due to forecasting weather and water conditions, monitoring and documentation of the catch, supervision and management, administrative record keeping and in part security. In search fisheries, information about the location of the stock can be made available to any number of boats, which can also be a prominent source of increasing returns to the number of boats $($ see $[1]) .^{3}$

The model also includes the specific and simplifying assumption that the harvest cost

\footnotetext{
3 [3] incorporates (globally) decreasing average costs by a harvest function that is (globally) concave in harvest and imposes a minimum average cost by an exogenous maximum harvest rate. In [15] and [16] average costs are U-shaped because of flow fixed costs that can be avoided if harvesting ceases completely.
} 
does not depend on the stock. In some fisheries, schooling behavior of the fish species has permitted the development of very effective means of harvesting such that with modern fishfinding equipment harvesting can be profitable even at low stock levels. Thus, changes in stock size may have little effect on harvest (see [17] for references to empirical studies that support this claim for specific fisheries). Analytically, the assumption will be useful because it can be seen that cyclical harvest policies are not caused by the presence of a positive externality from the stock of fish, as opposed to [19]. Alternatively put, we could easily incorporate the stock effect on the harvest cost, but this would make it much harder to see the link between increasing returns and cycles in harvesting.

Given that the steady state stock of fish $x=x^{*}$ is such that $F\left(x^{*}\right)=y^{*}<y^{\min }$, the stationary harvest rate $y^{*}$ is less than the minimum efficient scale, meaning that the fishery manager operates on the decreasing part of the average cost curve. Clearly, fishery's profit cannot be maximized by choosing a stationary harvest rate $y^{*}$ in the long-run. Instead, it is more efficient to adopt a harvest pattern where the harvest rate switches rapidly between zero and the minimum efficient scale $y^{\text {min }}$, while keeping the resource stock as close as possible to $x^{*}$. On average the harvest rate is then also equal to $y^{*}$, but costs are strictly less than $c\left(y^{*}\right)$ (see fig. 1). Such a policy is known as chattering control (for a more extensive description see ([3], pp. 144-152)). The use of chattering control implies that the corresponding trajectory cannot be termed 'optimal' in the usual sense of a payoff-maximizing path; the value of the objective can always be improved under any (well-defined) chattering control by switching a bit more rapidly (see [5]).

A harvest rate that switches infinitely fast between 0 and $y^{\text {min }}$ is not implementable and thus unrealistic. In practice, costs are present that prevent the occurrence of chattering harvest policies. In the literature (e.g., [10]) two kinds of costs are proposed in this respect: (1) start-up costs of a stock dimension incurred only when the control goes from zero to a 
positive level, or (2) adjustment costs of a flow dimension incurred whenever the control is changed. In [5] (theorem 2) it is shown that costs of type 1 do not generally rule out chattering controls if the scale economies arise from a cost function that has an initial concavity. The cost of type 2 are also suggested in [12], and they do generally rule out chattering. Thus, in our attempt to avoid chattering, we want to consider adjustment costs of type 2 .

Before introducing adjustment costs, let us briefly consider what happens if the resource stock does not equal its steady state value. This will most likely contribute to our understanding of managing a fish stock in practice, since in many fisheries the stocks are overfished and they are not in the neighborhood of a steady state. Using standard control theory (see, e.g., $[13])^{4}$, it is easy to establish that when $x(0)>x^{*}$ the fishery manager starts out by choosing a harvest rate $y \geq y^{\text {min }}$ such, that $x$ decreases and reaches the steady state level in finite time. If $x(0)<x^{*}$, harvesting will be ceased until the resource stock has increased to $x^{*}$.

\section{$3 \quad$ Fishery Model with Harvesting Adjustment Costs}

The analysis of the previous section is now extended by explicitly taking into account that it is costly to move resources to activities that change the harvest rate. These costs are made, e.g., in the process of hiring new fishermen or increasing the number of vessels (costs of type 2). To formulate the idea that the harvesting rate cannot be radically or instantaneously

\footnotetext{
${ }^{4}$ The optimality is verified by constructing an upper bound for the value function and showing that the harvesting policy actually generates a value function that approaches this upper bound. The upper bound is found by linearizing the concave part of the cost function (see fig. 1) and solving for the value function of this convexified model.
} 
adjusted, we consider the following problem:

$$
\begin{aligned}
& \max _{r} \int_{0}^{\infty}\{p y-c(y)-z(r)\} e^{-\rho t} d t \\
\dot{x}= & F(x)-y, x(0)=x_{0}>0, \\
\dot{y}= & r, y(0)=y_{0}>0, \\
x \geq & 0, y \geq 0, r \in[-d, d], 0<d<\infty .
\end{aligned}
$$

This extension (4)-(7) improves realism, because changes in the harvest rate are gradual. The decision maker's control, $r$ (e.g., investment in vessels or the hiring and firing of labor) determines through accumulation equation (6) the present harvest or catch $y$. A vector $(x(t), y(t), r(t))$ is called admissible when it satisfies (5)-(7) for all $t \geq 0$. To keep the resource management problem interesting, we consider only such admissible vectors. Thus, we assume, e.g., that the control set $[-d, d]$ is large enough such that it is in principle possible to control the system within the boundaries defined by (5)-(7).

The objective contains two types of flow costs. The first type is the harvesting cost $c(y)$, which arises as a consequence of performing harvesting effort. The second type arises due to the fact that, as in $([9]$, p. 364), the harvest $y$ can be adjusted only by incurring a cost $z(r) \equiv \frac{1}{2} \gamma r^{2}$. This cost structure makes the current harvest level and past adjustment costs explicitly dependent on each other, as opposed to [16]. The quadratic form simplifies the calculations but is not essential. The framework studied in [9] differs from ours, since in [9] only strictly concave optimal control problems are considered, where $x$ enters the objective function directly (also the fishery models in [17] and [19] belong to this class). [20] provides a general treatment for the occurrence of limit cycles in strictly concave optimal control models with two state variables. ${ }^{5}$

Because the harvest cost function is concave-convex, we cannot apply the Arrow- or

\footnotetext{
${ }^{5}$ The link between limit cycles and nonconvexities has been identified, e.g., in [10].
} 
Mangasarian-type sufficiency theorems (see [18]). However, in Appendices 1-2 we show the following result:

Proposition 1 There exists an optimal solution for problem (4)-(7). Moreover, given that

$$
p-c(y) / y>0 \quad \text { for } y<y_{s}
$$

holds, the solution satisfies $x(t) \rightarrow x^{*}(t)>0$ as $t \rightarrow \infty$, where $x^{*}(t)$ is an interior steady state or a periodic path for $x$.

The assumption (8), which will be maintained throughout the rest of the paper, implies that the surplus flow net of adjustments costs remains positive even when the fishery manager operates on the decreasing part of the average cost curve for fishing. If (8) did not hold, the manager would run a deficit by harvesting, for example, steady state quantities, which could call for abandoning the resource after a finite period of harvesting (see [15] $)^{6}$. By (8), we rule out this complication and ensure that the attention can be confined to long-run strategies characterized by an interior steady state or a periodic path ${ }^{7}$. A particular periodic path is a limit cycle. Also, as is shown in ([9], fig. 1), the steady state and limit cycle strategies cannot exist simultaneously, implying that if there exists a stable limit cycle, then the optimality of a (saddlepoint) stable steady state can be ruled out; if a stable steady state is encountered, then the optimality of the limit cycle can be ruled out. ${ }^{8}$

\footnotetext{
${ }^{6}$ Note that in an open-access fishery, assumption (8) could lead to the extinction of the stock.

${ }^{7}$ More precisely, the non-negativity conditions $x \geq 0$ and $y \geq 0$ together with the condition (8) imply that the fishery manager has an incentive to keep the stock positive, despite the fact that the stock does not directly enter the objective. The reason is that if $x$ equals zero, the harvest rate $y$ is reduced to zero, otherwise the constraint $x \geq 0$ is violated. However, $y=0$ is not optimal, since positive harvesting is profitable for any harvest rate below $y_{s}$.

${ }^{8}$ Strictly speaking, this holds for the cycle generated by the Hopf bifurcation and the steady state from which the cycle emerged when the bifurcation parameter is sufficiently near to the critical value. Thus, the
} 
To prove the existence of a limit cycle, we take the following steps. First, we derive a four-dimensional system of differential equations (canonical eqs.) out of the necessary optimality conditions for (4)-(7). The steady state stock of fish will be of equal size in both models (1)-(2) and (4)-(7). Second, we use the Hopf bifurcation theorem and the methods developed in [9] to study the existence of limit cycles for the system at hand.

To this end, let $L=H+\alpha x+\beta y$ be the Lagrangian for (4)-(7), where $H=p y-$ $c(y)-z(r)+\lambda(F(x)-y)+\mu r$ is the current value Hamiltonian. $(\lambda, \mu)$ are the co-state variables of the states $(x, y)$, and $(\alpha, \beta)$ are the current value multipliers for the constraints $x \geq 0, y \geq 0$, respectively ${ }^{9}$. Hence, $\mu$ gives the increase in the value of the objective due to an additional unit of harvest. Two contradictory mechanisms are at stake here. On the one hand, extra harvesting raises the fishery's revenue, but on the other hand this will lead to extra harvesting costs and a reduction of the stock of fish.

Since $L_{r r}=H_{r r}<0$, maximization of the Hamiltonian yields a unique solution. It follows ([8], corol. 6.2) that $r$ is continuous. The direct implication is that the time derivative of the harvesting rate is also continuous which precludes the harvesting rate from chattering.

Use the first order interior condition for $r$ to eliminate this control variable and derive conclusion is of a local nature. Also, besides the limit cycle, periodic path may be characterized by regularly occurring periods with $y=\dot{y}=0$ followed by periods in which $y>0$ and $\dot{y} \neq 0$. We were unable to rule out this possibility but neither could we find an example of it. However, despite this possibility proposition 1 holds.

${ }^{9}$ We follow ([14], theorem 10.3.1) in the formulation of the state constraints. 
the following canonical eqs. in $(x, y, \lambda, \mu)$ :

$$
\begin{aligned}
& \dot{x}=F(x)-y \\
& \dot{y}=\mu / \gamma \\
& \dot{\lambda}=\lambda\left(\rho-F^{\prime}(x)\right)-\alpha \\
& \dot{\mu}=\mu \rho-\left(p-c^{\prime}(y)-\lambda\right)-\beta,
\end{aligned}
$$

where (11)-(12) hold whenever $\dot{\lambda}$ and $\dot{\mu}$ exist. By (10), we have $\mu=0$ in a steady state. Furthermore, (8) implies that $p-c^{\prime}(y)>0$ for a steady state where $y<y^{\min }$. Since $\lambda>0$, it follows from (11) that in such an interior steady state $\rho=F^{\prime}(x)$, i.e. marginal growth of the stock of fish equals the discount rate. This is the well-known "golden rule" result when the objective function is independent of the stock (see, e.g., [3], p.151). The result differs from ([9], [19]), where the positive externality implied by the instantaneous positive valuation of the stock results in a growth rate that falls below the discount rate. Thus, as opposed to ([9], [19]), the positive externality from $x$ is not the source of limit cycles in our setting.

Taking into account that the stock of fish follows logistic growth with $F(x)=x(1-x)$, the interior steady state solution of (9)-(12) is:

$$
\begin{aligned}
& x^{*}=(1-\rho) / 2 \\
& y^{*}=\left(1-\rho^{2}\right) / 4 \\
& \lambda^{*}=p-c^{\prime}\left(y^{*}\right) \\
& \mu^{*}=0 .
\end{aligned}
$$

Clearly, the steady state stock $x^{*}$ is of the same size in both models (1)-(2) and (4)-(7). Also, the steady state harvest rate $y^{*}$ equals the time average of the chattering harvest rate described in section 2. By (8), $p-c^{\prime}\left(y^{*}\right)>0$ for $y^{*} \leq y^{\min }$, which ensures that the steady state shadow price of the fish stock $\lambda^{*}$ remains positive even when $y^{*}$ lies in the nonconvex region of the harvest cost function. 
To prove the existence of limit cycles for (9)-(12), let $\gamma$ be the bifurcation parameter (this is convenient because a change in $\gamma$ does not alter the steady state coordinates $\left.\left(x^{*}, y^{*}, \lambda^{*}, \mu^{*}\right)\right)$. Moreover, let $q=(x, y, \lambda, \mu)$ and $\dot{q}=f(q, \gamma)$ with $f\left(q^{*}, \gamma\right)=0$ where the components of $f$ are given by the right hand side of (9)-(12). We should see the system (9)-(12) as a family of nonlinear systems that is generated by varying the bifurcation parameter $\gamma$. The Hopf bifurcation theorem can be used to study the stability properties of this family of systems. The existence of limit cycles is ensured if for some value $\gamma=\gamma^{*}$ (i) the Jacobian $J\left(\gamma^{*}\right) \equiv \partial f\left(q^{*}, \gamma^{*}\right) / \partial q$ evaluated at the steady state $q^{*}$ has a pair of purely imaginary eigenvalues and if (ii) the imaginary axis is crossed at nonzero velocity. To check these two conditions we follow closely [9]. First, the eigenvalues $\omega_{i}(i=1, \ldots, 4)$ for the Jacobian $J\left(\gamma^{*}\right)$ are given by the following formula (see [7]):

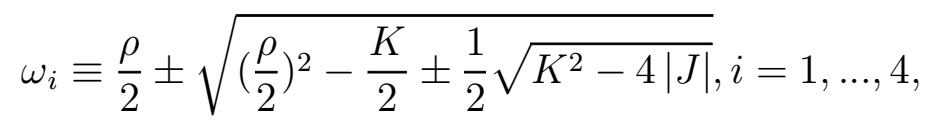

where

$$
K \equiv\left|\begin{array}{cc}
\frac{\partial \dot{x}}{\partial x} & \frac{\partial \dot{x}}{\partial \lambda} \\
\frac{\partial \dot{\lambda}}{\partial x} & \frac{\partial \dot{\lambda}}{\partial \lambda}
\end{array}\right|+\left|\begin{array}{cc}
\frac{\partial \dot{y}}{\partial y} & \frac{\partial \dot{y}}{\partial \mu} \\
\frac{\partial \dot{\mu}}{\partial y} & \frac{\partial \dot{\mu}}{\partial \mu}
\end{array}\right|+2\left|\begin{array}{cc}
\frac{\partial \dot{x}}{\partial y} & \frac{\partial \dot{x}}{\partial \mu} \\
\frac{\partial \dot{\lambda}}{\partial y} & \frac{\partial \dot{\lambda}}{\partial \mu}
\end{array}\right|,
$$

and the determinant of the Jacobian, $|J|$, are evaluated at the steady state. The Jacobian at the steady state is seen to be

$$
J=\left|\begin{array}{cccc}
\rho & -1 & 0 & 0 \\
0 & 0 & 0 & 1 / \gamma \\
2\left(p-c^{\prime}\left(y^{*}\right)\right) & 0 & 0 & 0 \\
0 & c^{\prime \prime}\left(y^{*}\right) & 1 & \rho
\end{array}\right| .
$$

Straightforward calculations give

$$
\begin{aligned}
|J| & =\frac{2\left(p-c^{\prime}\left(y^{*}\right)\right)}{\gamma} \\
K & =\frac{-c^{\prime \prime}\left(y^{*}\right)}{\gamma}
\end{aligned}
$$


The expression in (20) is positive when $\lambda^{*}>0$ which holds even when the harvest cost function is nonconvex at the equilibrium, i.e., when $y^{*}<y^{\mathrm{min}}$. If the harvest function is convex at the equilibrium, then $c^{\prime \prime}\left(y^{*}\right)>0$ and thus $K<0$. This together with $|J|>0$ implies saddlepoint stability (see, e.g., [9], fig. 1). As $c^{\prime \prime}\left(y^{*}\right)>0$ holds for $y_{s}<y^{*}=$ $\left(1-\rho^{2}\right) / 4$, the latter inequality is a sufficient condition for the saddlepoint stability. By Lemma 1 in [9], the existence of limit cycles requires that $|J|>0$ and $K>0^{10}$. The expression for $K$ is positive if and only if $c^{\prime \prime}\left(y^{*}\right)<0$, which holds when the steady state harvest $y^{*}$ falls below $y_{s}$. Recall that this condition was sufficient for the chattering harvest policy to emerge in section 2. Thus, the harvest policy can be characterized by a limit cycle only if the harvest is chattering in case adjustments are costless. ${ }^{11}$ Also note that, contrary to [9] and [19], in our framework the positive externality of the stock is not required for the positivity of $K$.

A pair of purely imaginary eigenvalues can emerge only when (see [9])

$$
|J|=\left(\frac{K}{2}\right)^{2}+\rho^{2} \frac{K}{2} .
$$

After substitution of the expressions (20)-(21) into (22), we can calculate the critical value $\gamma=\gamma^{*}$ explicitly:

$$
\gamma=\gamma^{*} \equiv \frac{c^{\prime \prime}\left(y^{*}\right)^{2}}{8\left(p-c^{\prime}\left(y^{*}\right)\right)+2 \rho^{2} c^{\prime \prime}\left(y^{*}\right)}>0
$$

where the inequality follows from $|J|>0$ and $K>0$. Thus, the first requirement of the Hopf bifurcation theorem is met when $\gamma=\gamma^{*}$. The second requirement is fulfilled if we can show that real parts of the eigenvalues vary strictly with the bifurcation parameter at the

\footnotetext{
${ }^{10}$ This holds since $K>0$ and $|J|>0$ are necessary conditions for two purely imaginary eigenvalues and, thus, for the emergence of the Hopf bifurcation.

${ }^{11}$ Contrastingly, in [10] the associated one-state-variable problem had to be of the saddlepoint type in order to establish cyclical strategies in the extended problem.
} 
bifurcation point, i.e. if

$$
\frac{d \operatorname{Re}\left(\omega_{i}\left(\gamma^{*}\right)\right)}{d \gamma} \neq 0
$$

To verify this we need to find an expression for the real parts. To this end, suppose (17) yields an eigenvalue with real and imaginary parts, $a+b j$, where $j$ denotes the imaginary number and where $a$ vanishes at the bifurcation point. In Appendix 3 we show that

$$
a \equiv \frac{\rho}{2}-\frac{1}{4} \sqrt{2 \rho^{2}-4 K+2 \sqrt{\rho^{4}-4 \rho^{2} K+16|J|}}
$$

and that

$$
\frac{d a}{d \gamma}=\frac{K c^{\prime \prime}\left(y^{*}\right)}{4 \rho \gamma^{* 2}\left[\rho^{2}+2 K\right]}<0
$$

This completes the proof of the existence of limit cycles.

Finally, let us briefly discuss what happens if the starting value of the resource stock is much larger or lower than the steady state level so that it cannot be part of the adjustment path to the limit cycle (assuming for the time being that the cycle is stable). In fact, what will happen is about the same as described at the end of section 2 , thus $y=0$ if $x$ is low and $y>y^{\text {min }}$ if $x$ is large. Presumably, this adjustment phase will last until a point on the adjustment path to the cycle is reached. Compared to the previous model, the harvest level will exhibit a more gradual movement over time because of the harvesting adjustment costs that occur in the present model.

\section{$4 \quad$ Stability Analysis}

Having established that a limit cycle may replace the steady state as a long run equilibrium, it is natural to ask whether this new 'steady state' is stable. Alternatively put, can the cyclical harvest policy be approached in the long-run for a given initial stock of fish and 
harvest level? If not, the limit cycle does not represent a generic long-run strategy. The analytical stability check of this type is a very tedious undertaking even for specific examples (see [9]). For this reason, in most economic applications only the two conditions ensuring the existence of a limit cycle (i.e., the Jacobian having a pair of purely imaginary eigenvalues, and the imaginary axis being crossed at nonzero velocity) are verified, but not its stability (see, e.g., [19]). Because our model has a parsimonious structure, we are able to derive an analytical expression for the stability parameter, denoted by $A$, which must be negative if the limit cycle policy represents a generic long-run policy. This stability condition can be derived for any given harvest cost function and, therefore, used to identify those cost structures that not only exhibit increasing returns but also lead to stable limit cycles. Following the steps reported in [9], the stability parameter is seen to be

$$
\begin{gathered}
A=\frac{w^{2} \rho\left[B_{1}\left(c^{\prime \prime}\left(y^{*}\right)\right)^{2}+B_{2} c^{\prime \prime}\left(y^{*}\right)+B_{3}\right]}{2\left(c^{\prime \prime}\left(y^{*}\right)\right)^{2} \rho^{2}\left(\rho^{2}+9 w^{2}\right)\left(4 w^{2}+\rho^{2}\right)}, \\
w=\sqrt{-c^{\prime \prime}\left(y^{*}\right) / 2 \gamma^{*}} \\
B_{1}=24\left(\rho^{2}+9 w^{2}\right)>0 \\
B_{2}=-6 c^{(3)}\left(y^{*}\right)\left(\rho^{4}+21 \rho^{2} w^{2}+28 w^{4}\right)+c^{(4)}\left(y^{*}\right)\left(\rho^{6}+12 \rho^{4} w^{2}+29 \rho^{2} w^{4}+18 w^{6}\right) \\
B_{3}=4\left(c^{(3)}\left(y^{*}\right)\right)^{2} w^{2}\left(\rho^{2}+w^{2}\right)\left(\rho^{2}+4 w^{2}\right)>0,
\end{gathered}
$$

where $c^{(i)}(y)$ denotes the derivative of order $i(>2)$. Although the expression (27) does not suggest an insightful economic interpretation, it can be used to articulate relatively simple restrictions on the harvest cost function. Note first that requiring $A<0$ amounts to requiring $B_{1}\left(c^{\prime \prime}\left(y^{*}\right)\right)^{2}+B_{2} c^{\prime \prime}\left(y^{*}\right)+B_{3}<0$. By the existence of the cycle, $c^{\prime \prime}\left(y^{*}\right)<0$, which implies that the stability condition can hold only if $B_{2}>0$. In view of the expression for $B_{2}$, stable limit cycle policies can be immediately ruled out when the harvest cost is characterized by $c^{(3)}(y)=c^{(4)}(y)=0$ or $c^{(3)}(y) \geq 0$ and $c^{(4)}(y) \leq 0$. Stable cycles are most likely to occur when $c^{(3)}(y)<0$ and $c^{(4)}(y) \geq 0$. Note that these requirements go beyond the assumption 
of increasing returns, $c^{\prime \prime}(y)<0$, which was a necessary condition for the existence of a limit cycle.

The occurrence of stable limit cycle and saddlepoint policies depends on the size of adjustment costs. To illustrate this, we next approximate the concave part of the harvest cost function as follows

$$
c(y) \equiv\left\{\begin{array}{c}
y-\frac{1}{2} y^{2}-\frac{1}{3} y^{3} \text { for } y \leq y_{s} \\
c\left(y_{s}\right)+C\left(y-y_{s}\right) \text { for } y>y_{s}
\end{array}\right.
$$

where $y_{s}<(\sqrt{5}-1) / 2$ and $C\left(y-y_{s}\right)$ is increasing and convex in $y$. Assuming that $y^{*}=$ $\left(1-\rho^{2}\right) / 4<y_{s}$, the steady state harvest falls into the nonconvex part of the cost function. Specifying the values for $p$ and $\rho$, the bifurcation point $\gamma^{*}$ and the stability parameter can be explicitly evaluated. For example, for $p=2$ and $\rho=.02, \gamma^{*} \approx .214$ and $A \approx-152.48$ Thus, this bifurcation point expels a stable cycle. Using the same parameters, fig. 2 plots condition (22) versus the parameter $\gamma$. Since $|J|$ and $K$ are always positive we know, e.g., from fig. 1 in [9] that the dynamic system exhibits saddlepoint stability (with damped cycles) if (22) holds with greater than sign, and two purely imaginary eigenvalues exist when $|J|$ equals $(K / 2)^{2}+\rho^{2} K / 2$. Stable limit cycles occur when $|J|$ is less than and sufficiently close to $(K / 2)^{2}+\rho^{2} K / 2$. Unstable spirals occur otherwise. Applying this knowledge to fig. 2 we conclude that large values of $\gamma$ lead to saddlepoint stability, while stable cyclical strategies exist for $\gamma<\gamma^{*}$ when $\gamma$ is sufficiently close to $\gamma^{*}$. This makes sense: a high value of $\gamma$ make changes in $y$ costly, indicating difficulties in adjusting the harvest rate and, thereby, dictating the optimality of a constant steady state harvest. A low value of $\gamma$ means easy adjustments of $y$ which, at least partly, can be used to convexify the harvest cost function. Thus, optimal cyclical strategies are conceivable when the benefits from convexification dominate the costs from cycling. 


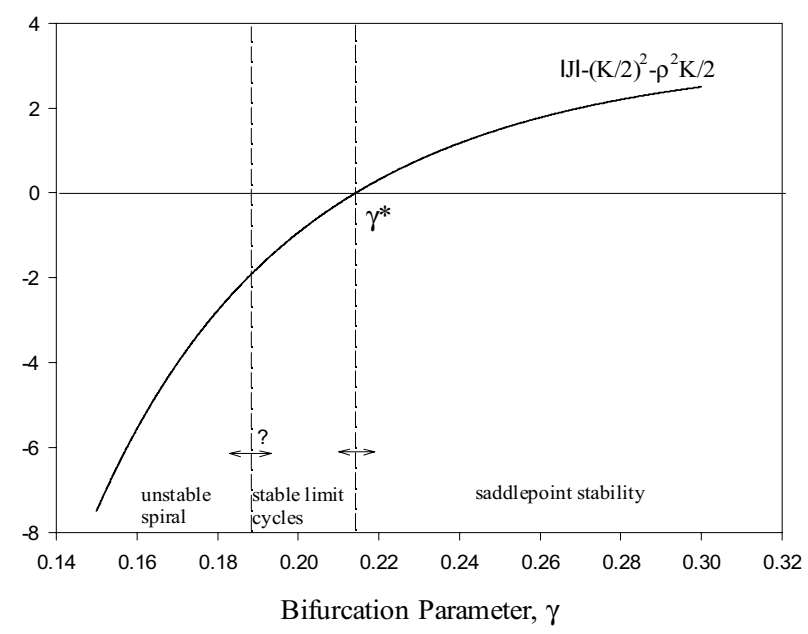

Figure 2: Stability Properties for Variations of $\gamma(p=2, \rho=.02)$

From fig. 2 it can also be concluded that for very low values of $\gamma$ unstable spirals occur. Although this result holds only locally, i.e., in the neighborhood of the steady state, it rules out both the limit cycle and steady state policies as optimal long-run policies. Note that the condition (8) rules out corner solutions where both the stock and harvest are reduced to zero in the long run (see also footnote 6). These observations lead to the following puzzle: for very low adjustment costs, the long-run harvest may not be stationary nor cyclical in the sense of an interior limit cycle. We conjecture that the solution to this puzzle is a periodic harvest characterized by regularly occurring periods with $y=\dot{y}=0$ followed by periods in which $y>0$ and $\dot{y} \neq 0$. Given the fact that for costless adjustments the optimal harvest level is jumping between zero and a positive level (see section 2), it is most likely that the optimal harvest is characterized by a periodic path also for positive but low values of $\gamma$. 


\section{Concluding Remarks}

In this paper the implications of the frequently occurring scale economies in fishing are analyzed within a theoretical framework. After an introductory analysis we developed an optimal control model in which it is costly to change the harvesting level. These costs arise due to hiring/firing workers, buying/selling boats, maintenance of temporarily inactive boats (mothballing), etc. Essentially, two types of long-run behavior are generated. First, large adjustment costs of changing harvesting levels lead to the occurrence of a long run equilibrium that is a saddle point. In this case, the harvest converges to a constant and stabilizes the stock of fish, meaning that the natural growth of the resource is neutralized by the harvest. Second, and more interesting, a scenario could be identified under which persistent oscillations occur. This means that changes in the harvesting level fluctuate persistently over time, implying that not only the harvest rate oscillates but also the stock of fish. In this case, periods with excessive harvesting occur, alternated by periods in which modest harvesting levels let the stock of fish recover.

It is a well-known fact that pulse fishing occurs regularly in practice ( $[3]$, p. 152). Our paper provides a theoretical explanation for this observed behavior by the linking the existence of limit cycles to increasing returns in fishing.

In the framework we considered it was assumed that the price of fish is fixed. On the other hand it could also be the case that prices decrease if the supply of fish increases, implying that the fishery has some market power. Intuitively it can be expected that taking market power into account will lead to a dampening of the harvest oscillations, since a high harvest rate implies that the supply of fish is also high which leads to a low price of fish and thus a lower incentive for harvesting. A low harvest rate induces a high price of fish and this will increase harvesting.

It is certainly admitted that the proposed model is highly stylized and probably much 
too simple to model a concrete planning situation. It shows, however, that under some circumstances alternating periods of high and low harvesting levels that reassure a sufficiently high level of the renewable resource have a clear economic meaning. Since we do not deal with a concrete planning case, no effort has been made at this stage to validate our model with empirical data. However, the latter task should clearly be a main issue for future research (cf. [17]).

\section{Appendix 1: Existence}

To prove the existence we apply the infinite horizon existence theorem 6.10 in [18]. First, our control set $U=[-d, d]$ is closed and bounded. Second, for all admissible $(x(t), y(t), r(t))$ the objective function is bounded above by

$$
\{p \bar{y}-c(\bar{y})\} e^{-\rho t} \geq\{p y-c(y)-z(r)\} e^{-\rho t} \equiv J,
$$

where $\bar{y}=\arg \max _{y}\{p y-c(y)\}$, and $\int_{0}^{\infty}\{p \bar{y}-c(\bar{y})\} e^{-\rho t} d t=\{p \bar{y}-c(\bar{y})\} / \rho<\infty$. Third, the set

$$
N(x, y, U, t)=\left\{\left(n_{0}, n_{1}, n_{2}\right)=[J+\zeta, F(x)-y, r]: r \in U, \zeta \leq 0\right\}
$$

is convex for any given $(x, y, t)$ because $J$ is a concave function of $r$ (see fig. 3). Fourth, for piecewise continuous, non-negative functions $a_{1}(t) \equiv 0, a_{2}(t) \equiv 0, b_{1}(t) \equiv F\left(x_{m s y}\right), b_{2}(t) \equiv d$, where $x_{m s y}=\arg \max _{x} F(x)$, the conditions

$$
\begin{aligned}
|F(x)-y| & \leq a_{1}(t) \times|x|+b_{1}(t) \\
|r| & \leq a_{2}(t) \times|y|+b_{2}(t)
\end{aligned}
$$

hold for all $(x, y, t)$. Fifth, clearly there exists an admissible vector $(x(t), y(t), r(t))$. Finally, because there are no mixed constraints, the other requirements of the theorem are trivially met. Q.E.D.

\section{Appendix 2: Implications of (8)}




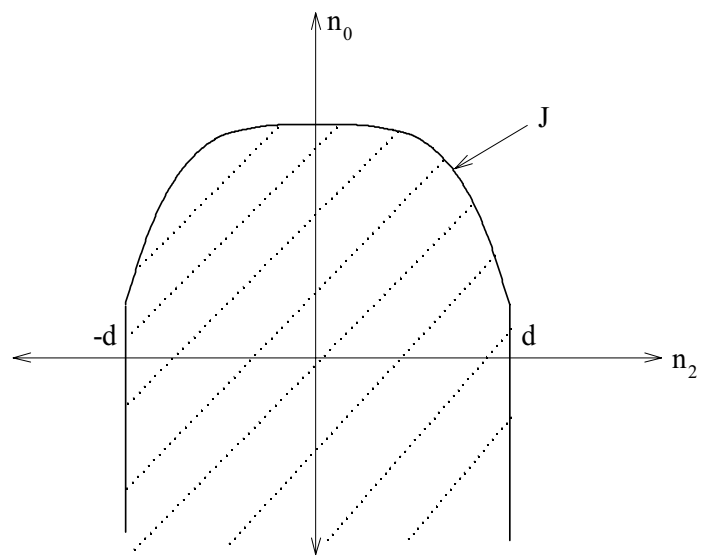

Figure 3: Convexity of the Set $N(x, y, U, t)$

Suppose $x(t)$ satisfies the necessary conditions for optimality but does not satisfy $x(t) \rightarrow$ $x^{*}>0$ as $t \rightarrow \infty$, where $x^{*}$ is a steady state or a periodic path for $x$. Then, because $F(0)=F(1)=0$ and $\dot{x}<0$ for $x>1$, there are four conceivable cases: (i) $x(t)=0$ for all $t \geq T^{1}<\infty$; (ii) $x(t) \rightarrow 0$ as $t \rightarrow \infty$; (iii) $x(t)=1$ for all $t \geq T^{2}<\infty$; and (iv) $x(t) \rightarrow 1$ as $t \rightarrow \infty$

Case (i). By $F(0)=0, y=\dot{y}=0$ for $t \geq T^{1}$. Then, conditions (10) and (12) imply that $\beta(t)=-\left(p-c^{\prime}(0)-\lambda\right) \geq 0$ for $t \geq T^{1}$. Hence, for $t \geq T^{1}$, we must have $\lambda \geq p-c^{\prime}(0)>0$, where the last inequality follows from (8). Using $\lambda>0, \rho-F^{\prime}(0)=\rho-1<0$ and $\alpha \geq 0$ in (11) yields $\dot{\lambda}<0$ for all $t \in\left[T^{1}, \infty\right)$. Thus, $\lambda<p-c^{\prime}(0)$ must hold for some $t$ in the interval $\left[T^{1}, \infty\right)$, which is a contradiction.

Case (ii). By $F(0)=0, y, \dot{y} \rightarrow 0$ as $t \rightarrow \infty$. Then, it must be the case that $\dot{\mu} \rightarrow 0$, i.e., $-\left(p-c^{\prime}(y)-\lambda\right) \rightarrow 0$ as $t \rightarrow \infty$. But this cannot hold, because, by (11) and $\alpha=0, \lambda$ cannot converge to $p-c^{\prime}(0)>0$.

Case (iii). By $F(1)=0$ and $\dot{x}=0$ for $t \geq T^{2}, y=\dot{y}=0$ for $t \geq T^{2}$. By (12), we must 
have $\beta(t)=-\left(p-c^{\prime}(0)-\lambda\right) \geq 0$, i.e. $\lambda>0$ for $t \geq T^{2}$. Using $\lambda>0, \rho-F^{\prime}(1)>0$ and $\alpha=0$ in (11) yields $\dot{\lambda}>0$ and $\ddot{\lambda}>0$ for $t \in\left[T^{1}, \infty\right)$. Thus, $\lambda \rightarrow \infty$ as $t \rightarrow \infty$, which contradicts the transversality condition $\lim _{t \rightarrow \infty} \lambda x=0$.

Case (iv). By $F(1)=0, y, \dot{y} \rightarrow 0$ as $t \rightarrow \infty$. Again, we obtain a contradiction by the arguments given in the case (ii). Q.E.D.

\section{Appendix 3: Expressions (25)-(26)}

By (17)

$$
\begin{gathered}
a+b j=\frac{\rho}{2} \pm \sqrt{\left(\frac{\rho}{2}\right)^{2}-\frac{K}{2} \pm \frac{1}{2} \sqrt{K^{2}-4|J|}}, \text { or } \\
\left(a+b j-\frac{\rho}{2}\right)^{2}=\left(\frac{\rho}{2}\right)^{2}-\frac{K}{2} \pm \frac{1}{2} \sqrt{K^{2}-4|J|} .
\end{gathered}
$$

Expand the left hand side of (29) and equate coefficients of the real parts which yields:

$$
a^{2}-b^{2}-\rho a+\frac{\rho}{4}^{2}=\frac{\rho}{4}^{2}-\frac{K}{2}
$$

and of the imaginary parts which, after squaring, yields

$$
b^{2}\left(-4 a^{2}+4 a \rho-\rho^{2}\right)=\frac{1}{4}\left(K^{2}-4|J|\right) .
$$

Using (30)-(31), we obtain a fourth degree equation for $a$,

$$
a^{4}-a^{3} 2 \rho+a^{2}\left(\frac{5}{4} \rho^{2}+\frac{1}{2} K\right)-a \rho\left(\frac{\rho^{2}}{4}+\frac{1}{2} K\right)+\left(\frac{K^{2}}{16}+\frac{\rho^{2} K}{8}-\frac{|J|}{4}\right)=0,
$$

which has the roots

$$
a_{i} \equiv \frac{\rho}{2} \pm \frac{1}{4} \sqrt{2 \rho^{2}-4 K \pm 2 \sqrt{\rho^{4}-4 \rho^{2} K+16|J|}}, i=1, \ldots, 4
$$

The root that vanishes at the bifurcation point is given by (25) because (22) implies that $a=\frac{\rho}{2}-\frac{1}{4} \sqrt{4 \rho^{2}}=0$. Differentiate (25) w.r.t $a$ and $\gamma$ :

$$
\frac{d a}{d \gamma}=\frac{-1}{8 \sqrt{2 \rho^{2}-4 K+2 \sqrt{\rho^{4}-4 \rho^{2} K+16|J|}}} \times\left[-4 \frac{d K}{d \gamma}+\frac{16 \frac{d|J|}{d \gamma}-4 \frac{d K}{d \gamma} \rho^{2}}{\sqrt{\rho^{4}-4 \rho^{2} K+16|J|}}\right] .
$$


Evaluating the derivative $\frac{d a}{d \gamma}$ at the bifurcation point $\gamma=\gamma^{*}$ yields

$$
\begin{aligned}
\frac{d a}{d \gamma} & =\frac{-1}{16 \rho} \times\left[-4 \frac{d K}{d \gamma}+\frac{16 \frac{d|J|}{d \gamma}-4 \frac{d K}{d \gamma} \rho^{2}}{\rho^{2}+2 K}\right], \\
& =\frac{1}{4 \rho \gamma^{* 2}} \times\left[c^{\prime \prime}\left(y^{*}\right)+\frac{8 \lambda^{*}+\rho^{2} c^{\prime \prime}\left(y^{*}\right)}{\rho^{2}+2 K}\right],
\end{aligned}
$$

where (35) is obtained by using (22), and (36) by using $\frac{d K}{d \gamma}=\frac{c^{\prime \prime}\left(y^{*}\right)}{\gamma^{* 2}}$ and $\frac{d|J|}{d \gamma}=\frac{-2\left(p-c^{\prime}\left(y^{*}\right)\right)}{\gamma^{* 2}}=$ $\frac{-2 \lambda^{*}}{\gamma^{* 2}}$. By $(23), 8 \lambda^{*}+\rho^{2} c^{\prime \prime}\left(y^{*}\right)=c^{\prime \prime}\left(y^{*}\right)^{2} \frac{1}{\gamma^{*}}-\rho^{2} c^{\prime \prime}\left(y^{*}\right)$, which can be substituted into (36) to obtain

$$
\begin{aligned}
\frac{d a}{d \gamma} & =\frac{c^{\prime \prime}\left(y^{*}\right)^{2} \frac{1}{\gamma^{*}}+2 K c^{\prime \prime}\left(y^{*}\right)}{4 \rho \gamma^{* 2}\left(\rho^{2}+2 K\right)} \\
& =\frac{K c^{\prime \prime}\left(y^{*}\right)}{4 \rho \gamma^{* 2}\left(\rho^{2}+2 K\right)}<0,
\end{aligned}
$$

where the last equality follows from the definition of $K$. Q.E.D.

\section{References}

[1] Björndal, T., J. Conrad, and K. Salvanes, Stock Size, Harvesting Costs, and the Potential for Extinction: The Case of Sealing, Land Economics 69, 156-167 (1993).

[2] Björndal, T., Production Economics and Optimal Stock Size in a North Atlantic Fishery, Scandinavian Journal of Economics 89, 145-164 (1987).

[3] Clark, C., Mathematical Bioeconomics, "The Optimal Management of Renewable Resources", New York, John Wiley \& Sons (1990).

[4] Clark, C., F. Clarke, and G. Munro, The Optimal Exploitation of Renewable Resource Stocks: Problems of Irreversible Investment, Econometrica 47, 25-47 (1979).

[5] Davidson, R. and R. Harris, Nonconvexities in Continuous-Time Investment Theory, Review of Economic Studies, XLVIII, 235-253 (1981). 
[6] Dawid, H., and M. Kopel, On the Economically Optimal Exploitation of a Renewable Resource: the Case of a Convex Environment and a Convex Return Function, Journal of Economic Theory 76, 272-297 (1997).

[7] Dockner, E., Local Stability Analysis in Optimal Control Problems with Two State Variables, in: G. Feichtinger, ed., "Optimal Control Theory and Economic Analysis" 2, 89-103 (1985), North-Holland, Amsterdam.

[8] Feichtinger, G, and R.F. Hartl, Optimale Kontrole Oekonomischer Prozesse: Anwendungen des Maximumprinzips in den Wirtschaftswissenschaften, de Gruyter, Berlin (1986).

[9] Feichtinger, G., A. Novak, and F. Wirl, Limit Cycles in Intertemporal Adjustment Models, Journal of Economic Dynamics and Control 18, 353-380 (1994).

[10] Feichtinger, G., and G. Sorger, Optimal Oscillations in Control Models: How Can Constant Demand Lead to Cyclical Production?, Operations Research Letters 5, 277$281(1986)$.

[11] Hanneson, R.,Fishery Dynamics: A North Atlantic Cod Fishery, Canadian Journal of Economics 8, 151-173 (1975).

[12] Jorgensen, S., and P. Kort, Optimal Dynamic Investment Policies Under ConcaveConvex Adjustment Costs, Journal of Economic Dynamics and Control 17, 153-180 (1993).

[13] Jorgensen, S., and P. Kort, Optimal Investment and Finance in Renewable Resource Harvesting, Journal of Economic Dynamics and Control 21, 603-630 (1997).

[14] Leonard, D. and N. Van Long, "Optimal Control and Static Optimization in Economics", Cambridge University Press (1992). 
[15] Lewis, T. and R. Schmalensee, Nonconvexity and Optimal Exhaustion of Renewable Resources, International Economic Review 18, 535-552 (1977).

[16] Lewis, T. and R. Schmalensee, Nonconvexity and Optimal Harvesting Strategies for Renewable Resources, Canadian Journal of Economics XII, 677-691 (1979).

[17] Novak, A., V. Kaitala, and G. Feichtinger, Resource Leasing and Optimal Periodic Capital Investments, ZOR - Mathematical Methods of Operations Research 42, 47-67 (1995).

[18] Seierstad, K. and A. Sydsaeter, "Optimal Control Theory with Economic Applications", Amsterdam, North Holland (1987).

[19] Wirl, F., The Cyclical Exploitation of Renewable Resource Stocks May Be Optimal, Journal of Environmental Economics and Management 29, 252-261 (1995).

[20] Wirl, F., Pathways to Hopf Bifurcations in Dynamic Continuous-Time Optimization Problems, Journal of Optimization Theory and Applications 91, 299-320 (1996). 